\title{
Effets de la distance de prélèvement par rapport au pied de Eucalyptus camaldulensis dehn sur les paramètres physico-chimiques du sol en zone sud- soudanienne côtière (Sénégal -Base Casamance)
}

\author{
Arfang Ousmane Kémo Goudiaby, \\ Siré Diedhiou, PhD \\ Paul Diouf,
}

Université Assane SECK de Ziguinchor, Faculté des Sciences et Technologies, Laboratoire d'Agroforesterie et d'Ecologie,

Département d'Agroforesterie, Sénégal

Ibrahima Ndoye, PhD

Université Cheikh Anta Diop de Dakar, Faculté des Sciences et Technologies, Département de Biologie Végétale, Sénégal

Doi: 10.19044/esj.2019.v15n6p119 URL:http://dx.doi.org/10.19044/esj.2019.v15n6p119

\section{Résumé}

Les effets des arbres sur les propriétés physico-chimiques du sol sont très variables d'une espèce à une autre. L'objectif de ce travail est de contribuer à l'évaluation de l'effet de la distance de prélèvement par rapport au pied de E. camaldulensis sur les propriétés physico-chimiques du sol. Pour ce faire, des prélèvements de sol ont été effectués dans trois sites différents: Oussouye, Boukitingho et Boucotte Diembéring, à l'horizon 0-20 cm. Ces prélèvements ont été faits sur différentes distances par rapport à $E$. camaldulensis: à $0 \mathrm{~m}, 10 \mathrm{~m}, 20 \mathrm{~m}$ et à $30 \mathrm{~m}$ du pied. Quatre arbres ont été choisis au hasard par site. Sur chaque arbre, un (1) échantillon composite a été formé par distance suivant l'orientation Est-Ouest, Nord-Sud. Le facteur étudié a été la distance de prélèvement par rapport à E. camaldulensis. Au total 48 échantillons de sol ont été prélevés dans les trois sites. Ces échantillons ont été analysés au laboratoire de l'Institut National de Pédologie. L'analyse des résultats a montré que la distance de prélèvement par rapport à $E$. camaldulensis n'a pas d'effets significatifs sur les paramètres physiques du sol, quel que soit le site $(\mathrm{P} \geq 0,37)$. La texture du sol est sablo-limoneuse. Un $\mathrm{pH}$ relativement acide a été noté quel que soit le traitement. Aucune différence significative n'a été notée entre le carbone et la matière organique mesurés avec respectivement $\mathrm{P} \geq 0,19$ pour le carbone et $\mathrm{P} \geq 0,14$ pour la matière organique. Il ressort des analyses chimiques, que la distance par rapport à $E$. 
camaldulensis n'a pas d'effets significatifs sur les propriétés chimiques du sol. Les caractéristiques chimiques du sol à différentes distances de $E$. camaldulensis ne semblent pas être influencées par l'espèce, quelle que soit la distance et quel que soit le site $(\mathrm{P}>0,05)$.

Mots-clés: Propriété physique, Propriété Chimique, Eucalyptus camaldulensis, distance

\title{
Effects of the sampling distance from the foot of Eucalyptus camaldulensis Dehn on the physical- chemical parameters of the soil in the South Sudanese coastal zone (Senegal - Base Casamance)
}

\section{Arfang Ousmane Kémo Goudiaby, Siré Diedhiou, PhD \\ Paul Diouf,}

Université Assane SECK de Ziguinchor, Faculté des Sciences et Technologies, Laboratoire d'Agroforesterie et d'Ecologie, Département d'Agroforesterie, Sénégal

\section{Ibrahima Ndoye, PhD}

Université Cheikh Anta Diop de Dakar, Faculté des Sciences et Technologies, Département de Biologie Végétale, Sénégal

\begin{abstract}
The effects of trees on soil physical and chemical properties are very variable depending on species. The aim of this work is to evaluate the effect of Eucalyptus camaldulensis on soil physical and chemical properties. For that, soil samples were taken from 0 to $20 \mathrm{~cm}$ from the surface in three different sites: Oussouye, Boukitingho and Boucotte Diembéring. These soil samples were taken from various distances $(0,10,20$ and $30 \mathrm{~m})$ from E. camaldulensis trees. Four trees of $E$. camaldulensis were randomly chosen by site. A round each tree, a composite sample was made at each distance level. A total of 48 composite samples were taken in the three sites. These samples were taken to the laboratory of National Institute of Pedology for the analyses to determine the soil physical and chemical properties (texture, $\mathrm{pH}$, carbone and organic matter). The studied factors were the distance of sampling from $E$. camaldulensis and the species. The distance of sampling had no significant effects $(\mathrm{P}=0.37)$ on the soil physical parameters. The soil texture was sandy silt. The soil $\mathrm{pH}$ was relatively acid. There was no significant difference
\end{abstract}


( $>0.05)$ between soil carbon and organic matter content. The sampling distance had no significant effects $(\mathrm{P}>0.05)$ on the soil chemical properties. The chemical characteristics of the soil from different distances from $E$. camaldulensis were not influenced by the species.

Keywords: Physical property, chemical property, Eucalyptus camaldulensis, distance

\section{Introduction}

La réhabilitation des espaces dégradés a suscité beaucoup d'intérêt ces dernières années. Celle-ci est toujours considéré comme une approche efficace pour atténuer l'augmentation du $\mathrm{CO}_{2}$ atmosphérique (GIEC, 2007). Cette politique de reboisement a permis l'expansion rapide des espèces exotiques, comme Eucalyptus en Afrique, et au Sénégal.

En Casamance, l'espèce Eucalyptus camaldulensis a été plantée en masse en 2008 par le programme Bois Energie du Sénégal (BES), dans le cadre du projet BES. Ce remplacement de la végétation naturelle par une essence forestière exotique aurait des effets immédiats sur le sol, à travers des apport à la surface du sol d'une litière de composition particulière que la microflore endémique n'est pas habituée à dégrader. Ainsi, le premier stade de son influence sur le sol va être lié aux processus de décomposition et d'incorporation (Soumaré, 2012). Les apports quantitatifs et qualitatifs de litières conditionnent la qualité du sol, de même que l'abondance et la diversité de ses communautés microbiennes (Orwin et al., 2005, Bréchet et al., 2009). La qualité des sols peut être altérée par l'introduction d'essences exotiques qui par leurs besoins élevés, par leurs retombées organiques (pauvres en éléments minéraux ou difficilement dégradables par la microflore endémique) ou par leurs effets allélopathiques sur les espèces locales, finissent par appauvrir le sol. La litière des Eucalyptus est souvent citée comme riche en composés phénoliques inhibiteurs (Soumaré, 2012). Les Eucalyptus par leur matière organique, leurs racines (action mécanique) et leurs exsudations racinaires modifieraient les propriétés physico-chimiques du sol tant au niveau microporosité que macroporosité (Keller et Zengler, 2004, Farley et al., 2008). La nature du sol pourrait donc varier au fur et à mesure que l'on s'éloigne du tronc de l'arbre. Toutefois, peu d'études se sont intéressées sur les effets de l'espèce sur les propriétés physico-chimiques du sol en suivant le gradient de distance par rapport à l'arbre. Le présent travail se propose de contribuer à l'évaluation de l'effet de l'éloignement par rapport au pied de $E$. camaldulensis sur les propriétés physico-chimiques du sol. 


\section{Matériel et Méthodes}

\section{Présentation de la zone de l'étude}

Le sol utilisé pour ces analyses provient des plantations de $E$. camaldulensis âgées de 8 ans et situées dans les localités de Oussouye, Boukitingho et Boucotte Diembéring, localisées en zone soudano-côtier au Sud du Sénégal (Sagna, 2005). Cette zone est caractérisée par neuf mois de saison sèche et trois mois de saison pluvieuse, avec une moyenne pluviométrique de $1316 \mathrm{~mm}$. Les températures moyennes sont comprises entre $20^{\circ} \mathrm{C}$ et $35^{\circ} \mathrm{C}$. Par ailleurs, les vents dominants sont l'alizé et la mousson (Badji, 2016).

\section{Echantillonnage du sol}

Le prélèvement du sol a été effectué sur trois sites différents (Oussouye, Boukitingho et Boucotte Diembéring) à l'horizon 0-20 cm (Saidou, 2018). Ces prélèvements ont été faits à différentes distances par rapport à au pied de E. camaldulensis: $0 \mathrm{~m}, 10 \mathrm{~m}, 20 \mathrm{~m}$ et $30 \mathrm{~m}$ du pied de $E$. camaldulensis. Quatre arbres ont été choisis au hasard par site. Sur chaque arbre, un (1) échantillon composite a été formé par rapport à la distance suivant l'orientation Est-Ouest, Nord-Sud. Le facteur étudié a été la distance de prélèvement par rapport au pied de E. camaldulensis. Au total 48 échantillons de sol ont été prélevés sur les trois sites soit 4 x4x 3=48.

\section{Analyses de sol \\ Préparation des échantillons}

Les échantillons sont séchés à 1'ombre puis tamisés à $2 \mathrm{~mm}$ afin d'éliminer les éléments grossiers et les débris divers. Une partie de la terre fine (2 mm) a été broyée (sans détruire les particules du sol) et tamisée à $0,5 \mathrm{~mm}$ pour l'analyse des éléments totaux.

\section{Méthodes d'analyse}

Les analyses chimiques ont été effectuées au laboratoire de l'institut Nationale de Pédologie (INP). Les éléments chimiques analysés sont: la matière organique $(\mathrm{MOS})$, le $\mathrm{pH}$ (eau et $\mathrm{KCl}$ ), le carbone $(\mathrm{C})$ «total », l'azote $(\mathrm{N})$ «total », le phosphore $(\mathrm{P})$ « total» et « assimilable» et les bases échangeables.

\section{Dosage du pH}

Le pH (eau et KC1) du sol a été mesuré sur une suspension de solution par la méthode électrométrique au $\mathrm{pH}$-mètre à électrode de verre. Pour ce faire, $20 \mathrm{~g}$ de terre tamisée à $2 \mathrm{~mm}$ a été ajoutée à $50 \mathrm{ml}$ d'eau distillée puis agitée (la solution) pendant une heure à l'agitateur magnétique. La solution a été ensuite laissée au repos pendant une heure et le $\mathrm{pH}$ eau a été lu avec un pH- 
mètre étalonné à 7 et à 4. Après la mesure du $\mathrm{pH}$ eau, 3,7 g de $\mathrm{KCl}$ (réactif) a été rajouté à la solution de sol et le tout agité pendant $30 \mathrm{mn}$ avant la lecture du $\mathrm{pH} \mathrm{KCl}$.

\section{Dosage de l'azote total}

L'azote est dosé par la méthode Kjeldahl modifiée (Hillebrand et al., 1953). Les échantillons de sol ont été soumis à une minéralisation Kjeldahl, avec l'acide sulfurique et acide salicylique $\left.\left(\mathrm{C}_{7} \mathrm{H}_{6} \mathrm{O}_{3}\right]\right)$ en présence du peroxyde d'hydrogène $\left(\mathrm{H}_{2} \mathrm{O}_{2}\right)$ et du sélénium $(\mathrm{Se})$ utilisé comme catalyseur. Après la minéralisation, la solution aqueuse est filtrée au papier «Whatman» $125 \mathrm{~mm}$. Une partie du substrat a été ensuite prélevée et distillée. L'azote organique à l'issu de l'attaque est converti en azote ammoniacal $\left(\mathrm{NH}^{4+}\right)$ qui est déplacé de la solution par distillation. Les quantités d'azote déplacées ont été ensuite recueillies dans une solution d'acide borique (H3B03) qui a été dosées en retour à l'acide sulfurique $\left(\mathrm{H}_{3} \mathrm{SO}_{4}\right)$.

Le taux d'azote a été évalué en appliquant le taux de refus comme facteur de correction à travers la formule: $\% \mathrm{~N}=\% \mathrm{~N}$ mesuré $\mathrm{x}$ Taux de refus.

\section{Dosage du carbone total}

La méthode Walkley-Black a été utilisée (Nelson et Sommers, 1975). Elle consiste en une oxydation à froid d'un échantillon (solide ou liquide) par une solution de bichromate de potassium $\left(\mathrm{K}_{2} \mathrm{Cr}_{2} \mathrm{O}_{7}\right)$ en présence d'acide sulfurique $\left(\mathrm{H}_{2} \mathrm{SO}_{4}\right)$. L'excès de bichromate est dosé en retour avec une solution standard de $\mathrm{Fe}^{2+}$ (dans du sulfate d'ammonium ferreux: sel de Mohr dont la formule chimique est FeS04 $\left.\left(\mathrm{NH}_{4}\right)_{6}\right)$ pour déterminer la quantité qui a réagi.

\section{Dosage de phosphore total}

Le phosphore total a été mesuré sur le condensé de la minéralisation (BU.NA.SOLS, 1987). Pour ce faire, une solution d'acide ascorbique associé au molybdate d'ammonium et de l'eau distillée a été ajoutée à la solution minéralisée puis agitée à l'agitateur électrique. Le spectro-photomètre à UV/ visible standardisé a servi à la lecture directe des résultats.

\section{Dosage du phosphore assimilable}

La méthode Brey 1 (Bray and Kurtz, 1945) a été utilisée pour le dosage du phosphore assimilable L'acide chlorhydrique est utilisé pour extraire les formes de phosphore $(\mathrm{P})$ solubles dans l'acide. Le fluorure d'ammonium dissout les phosphates de $\mathrm{Ca}$, de $\mathrm{Fe}$ et d'Al en formant un complexe entre ces ions et ceux des métaux en solutions acide. Le dosage a été ensuite fait par colorimétrie en utilisant l'acide ascorbique qui réduit le complexe phosphomolybdique formé par ajout de molybdate d'ammonium en bleu de molybdène. La réaction est effectuée en présence d'acide sulfurique et la 
lecture effectuée avec un spectro-photomètre à UV visible. Un tamis $100(0,2$ $\mathrm{mm}$ ) a été utilisé pour tamiser le sol finement.

\section{Matière organique}

La procédure consiste à peser $1 \mathrm{~g}$ et à introduire la prise d'essai dans un ballon en Pyrex avec réfrigérant ascendant, puis à ajouter $10 \mathrm{ml}$ de solution de bichromate de potassium à $8 \%$ (exactement) et $15 \mathrm{ml}$ d'acide sulfurique concentré. Le produit est porté à ébullition douce. Au début de l'ébullition, chronométrer $5 \mathrm{mn}$, et laisser refroidir. Après cette étape, il faut ajouter $25 \mathrm{ml}$ d'eau distillée puis remuer le tout, ensuite prélever $10 \mathrm{ml}$ d'une partie aliquote à verser dans un bêcher de $250 \mathrm{ml}$ et à diluer avec $20 \mathrm{ml}$ d'eau distillée. Après cela, on n'ajoute $0,5 \mathrm{~g}$ de fluorure de sodium $\mathrm{NaF}$ en poudre par ml d'acide sulfurique présent soit 5 à $7 \mathrm{~g}$ (ou $10 \mathrm{ml}$ d'acide phosphorique $\mathrm{d}=1,71$ ). Ajouter à nouveau trois (3) gouttes de diphénylamine, puis la solution de sel de Mohr est versée dans une burette et l'excès de bichromate est titré. La couleur passe alors du bleu foncé au bleu vert.

\section{Bases échangeables}

Le procédé consiste à peser $20 \mathrm{~g}$ de terre dans un bêcher de $250 \mathrm{ml}$, ajouter $50 \mathrm{ml}$ d'acétate d'ammonium puis à agiter et laisser en contact une nuit. Le contenu est filtré le lendemain sur filtre lent en recueillant le filtrat dans une fiole jaugée de $250 \mathrm{ml}$. Ensuite Poursuivre la percolation par fraction successives de $50 \mathrm{ml}$ d'acétate d'ammonium qu'on met dans le bêcher en contact avec du sol pendant $30 \mathrm{mn}$, puis le contenu est agité et filtré. A la dernière fraction, verser la terre contenue dans le bêcher sur l'entonnoir en rinçant le bêcher. La solution est ajustée avec de l'acétate. Le filtre contenant le sol est remis dans le bêcher pour la mesure de la capacité d'échange. Après on procède au prélèvement de $20 \mathrm{ml}$ pour déterminer $\mathrm{Ca}^{++}$et $20 \mathrm{ml}$ pour $\mathrm{Mg}^{+}$.

\section{Traitement et analyse des données}

Le traitement et l'analyse des données ont été faits à l'aide du logiciel $\mathrm{R}$ version 3.4.2. Les moyennes des variables ont été comparées en utilisant le test de Tukey, au seuil de probabilité $\mathrm{p}=5 \%$ dans le cas où il $\mathrm{y}$ a un effet significatif $(\mathrm{p}<5 \%)$.

\section{Normes d'interprétation des données pédologiques}

L'analyse des sols a été réalisée avec l'aide des normes d'interprétations de données pédagogiques utilisées dans les pays tropicaux.

\section{Texture des sols}

La texture des différents échantillons de sol est déterminée à l'aide du triangle textural de Tavernier et Maréchal (1958) cité par Richer de Forges et 
al. (2008). L'approche méthodologique de détermination de la texture d'un échantillon de sols consiste, selon Masson (2012), à porter sur les trois axes du triangle les pourcentages d'argile, de limon et de sable en commençant par l'élément présentant le plus faible pourcentage. Pour chacun des points ainsi trouvés, il conviendra ensuite de dessiner une parallèle à l'axe précédent. L'intersection de ces trois parallèles désigne la classe texturale du sol analysé.

\section{Potentiel hydrogène $(\mathrm{pH})$}

La mesure du $\mathrm{pH}$ (potentiel hydrogène) d'un sol permet de définir son statut acido-basique. Pour la plupart des sols on note des valeurs comprises entre 4 et 8,5. La légende des séries de sol proposée par le Bureau Pédologie du Sénégal (1992) a été utilisée pour l'interprétation du pH (Tableau 1).

Tableau 1: Les normes d'interprétation du statut acido-basique des sols

\begin{tabular}{ll}
\hline Degré & Mesure du pH \\
\hline \hline Extrêmement acide & $<4,5$ \\
Très acide & $4,6-5,2$ \\
Acide & $5,3-5,5$ \\
Modérément acide & $5,6-6,0$ \\
Légèrement acide & $6,1-6,6$ \\
Neutre & $6,7-7,2$ \\
Légèrement alcalin & $7,3-7,9$ \\
Alcalin & $8,0-8,5$ \\
Très alcalin & $>8,5$ \\
\hline \hline
\end{tabular}

Source : Bureau Pédologie du Sénégal (1992)

\section{Carbone (C), Matière organique (MO) et Azote (N)}

La plupart des propriétés physico-chimiques des sols nécessaires à la croissance des plantes, à la stabilité de la structure du sol et à une aération suffisante dépendent de la qualité de la matière organique (MO) qu'ils comportent. Le taux de la Matière organique d'une fraction de sol est fonction de sa teneur en carbone organique (C) et est estimé en multipliant le pourcentage de ce carbone par le facteur 1,724 (Walkley et Black., 1934). En milieu soudanien et sahélien, le taux de Matière organique qui traduit un bon niveau de fertilité du sol, est estimé à 1,5 voire plus de $2 \%$.

L'activité microbienne, fortement influencée par les conditions micro climatiques, induit d'importantes transformations de l'azote $(\mathrm{N})$ du sol. Le rapport $\mathrm{C} / \mathrm{N}$ est un bon paramètre pour apprécier l'activité biologique du sol (Ramade, 2008). Les valeurs optimales de $\mathrm{C} / \mathrm{N}=10$ à 12 . Le $\mathrm{C} / \mathrm{N}$ élevé (15 à 20) traduit une activité biologique faible dans le sol. C'est-à-dire que le processus de minéralisation de la Matière organique est lent. Ce qui pourrait avoir comme conséquences une faible libération de l'azote disponible dans le sol (Masson, 2012). Par contre un rapport $\mathrm{C} / \mathrm{N}$ faible (4 à 12), rend compte d'une activité biologique intense, d'un processus de minéralisation rapide et d'une bonne libération de la quantité d'azote dans sol. L'échelle 
d'interprétation de la teneur de ces trois éléments ( $\mathrm{C}, \mathrm{MO}$ et $\mathrm{N})$ utilisée dans cette étude est celle présentée conjoitement en 2002 par le CIRAD et le GRET (2002) (Tableau 2).

Tableau 2: Estimations générales de $\mathrm{N}$ et $\mathrm{MO}$ pour des sols présentant 15 à $50 \%$ de teneurs en argile et limon

\begin{tabular}{lc}
\hline Estimation de : & Teneur en \% \\
\hline \hline Azote (N) & \\
Très pauvre & $0,10-0,25$ \\
Pauvre & $0,25-0,45$ \\
Moyen & $0,45-0,80$ \\
Riche & $0,80-1,50$ \\
Très riche & $1,50-3,00$ \\
Excellent & $3,00-6,00$ \\
& \\
Matière Organique (MO) & \\
Très pauvre & \\
Pauvre & $0,17-0,43$ \\
Moyen & $0,43-0,76$ \\
Riche & $0,76-1,35$ \\
Très riche & $1,35-2,55$ \\
Excellent & $2,55-5,10$ \\
\hline \hline & $5,10-10,10$ \\
\hline
\end{tabular}

\section{Phosphore (P) du sol}

L'analyse et l'interprétation de la teneur du phosphore assimilable des sols ont été effectuées suivant deux méthodes liées au pH. Pour les sols dont le $\mathrm{pH}$ est inférieur à 7,5, on utilise la solution d'extraction Bray 1 et pour les sols dont le $\mathrm{pH}$ est supérieur ou égal à 7,5, on utilise la solution d'extraction Olsen. Suivant la teneur en phosphore, la réponse à l'engrais diffère. La norme de Landon 1984 est souvent utilisée pour l'interprétation du phosphore (Tableau 3).

Tableau 3: Echelle d'estimation du Phosphore assimilable

\begin{tabular}{|c|c|c|c|}
\hline \multirow[b]{2}{*}{ Méthodes } & \multicolumn{3}{|c|}{ Estimations générales de la teneur du phosphore disponible en ppm } \\
\hline & $\begin{array}{c}\text { Bas (réponse aux engrais } \\
\text { très probable) } \\
\end{array}$ & $\begin{array}{c}\text { Moyen (réponse aux engrais } \\
\text { probables) } \\
\end{array}$ & $\begin{array}{l}\text { Haut (engrais } \\
\text { non probable) } \\
\end{array}$ \\
\hline OLSEN (ppm) & $<05$ & $02-15$ & $>15$ \\
\hline BRAY 1 (ppm) & $<15$ & $15-50$ & $>50$ \\
\hline
\end{tabular}

Source : Landon (1984)

\section{Calcium (Ca)}

Le calcium a un rôle extrêmement important dans la constitution des tissus végétaux et permet aux plantes de mieux se développer (Petit et Jobin, 2005). Sa carence ne s'observe que dans les sols ayant une capacité d'échange cationique basse aux valeurs des $\mathrm{pH}$ de 5,5 au moins. Il peut se produire également des carences en calcium quand le $\mathrm{pH}$ est élevé ainsi que lorsque la 
teneur en sodium est excessive. Si le sol a un calcium échangeable inférieur à $0,2 \mathrm{meq} / 100 \mathrm{~g}$, on observe une réponse très probable aux engrais calciques.

\section{Magnésium (Mg)}

Dans les pays tropicaux, le seuil de carence du magnésium se situe à 0,5 meq/100g. L'échelle de Landon (1984) est souvent utilisée comme norme d'interprétation de la teneur de cet élément chimique dans les sols (Tableau 4).

\begin{tabular}{|c|c|c|}
\hline \multirow{2}{*}{ Estimation } & Teneur en : & Teneur en : \\
\hline & Meq/100g & PPM \\
\hline Bas & $<0,2$ & $<30$ \\
\hline Moyen & $0,2-0,5$ & $30-60$ \\
\hline Haut & $>0,5$ & $>60$ \\
\hline
\end{tabular}

Source : Landon, 1984

\section{Potassium (K)}

Le potassium joue un rôle majeur dans la capacité des plantes à résister au stress induit (sécheresse, attaques de parasites, ...). Il intervient dans l'activation de leurs enzymes, la production de protéines et la photosynthèse (COMIFER, 2009). On observe une réponse probable avec les engrais potassiques si le potassium échangeable est inférieur à $0,2 \mathrm{meq} / 100 \mathrm{~g}$ et non probable si le $\mathrm{K}^{+}>0,5 \mathrm{meq} / 100 \mathrm{~g}$. Ces limites ne doivent pas être considérées comme définitives étant donné qu'elles sont soumises aux variations de la nature du sol, de l'environnement et de la plante.

\section{Sodium (Na)}

La présence du sodium en grandes quantités dans le sol peut être préjudiciable aux plantes et également agir sur les conditions physiques du sol. Les sols ayant du sodium échangeable supérieur à $1 \mathrm{meq} / 100 \mathrm{~g}$ sont considérés comme potentiellement sodiques (Landon, 1984).

\section{Capacité d'Echange Cationique (CEC.)}

La CEC d'un sol représente la quantité de cations (calcium, potassium, magnésium, sodium et ammonium) qu'il peut retenir sur son complexe argilohumique à un pH donné (Masson, 2012). La valeur de la CEC d'un sol est fonction de sa richesse en argile et en matière organique. Le calcul de la valeur de la CEC donne une bonne idée des teneurs en éléments cationiques échangeables $\left(\mathrm{K}^{+}, \mathrm{Ca}^{2+}, \mathrm{Mg}^{2+,} \mathrm{Na}^{+}\right)$et, donc, potentiellement disponibles pour les cultures. Le niveau de la CEC permet de préciser les doses et fréquences d'apports pour une fertilisation potassique et magnésienne (Tableau 5). 
Tableau 5: Normes d'interprétation de la CEC

\begin{tabular}{lc}
\hline Estimation & CEC (meq/100g) \\
\hline \hline Très haut & $>40$ \\
Haut & $25-40$ \\
Moyen & $15-25$ \\
Bas & $05-15$ \\
Très bas & $<5$ \\
\hline \hline
\end{tabular}

Source : Landon, 1984

\section{Résultats}

Effet de la distance de prélèvement par rapport au pied de Eucalyptus camaldulensis sur les propriétés physiques du sol

La teneur en argile est comprise entre $7,9 \%$ et $13,7 \%$ pour tous les traitements confondus. Cette teneur ne différe pas de façon significative quelle que soit la distance de prélèvement, par rapport au pied de E. camaldulensis $(\mathrm{P} \geq 0,07)$ (Tableau 6). Cependant, cette teneur varie différemment suivant les sites d'étude. L'analyse de variance a révélé que la teneur en'argile mesurée à $0 \mathrm{~m}$ de E . camaldulensis à Oussouye et à Boukitingho, a été significativement plus élevée que celle obtenue à Boucotte Diembéring $(\mathrm{P}<0,02)$.

Pour ce qui est des limons fins, leur teneur varie de $1,8 \%$ à $4,7 \%$ pour tous les traitements. L'ANOVA n'a révélé aucune différence significative entre les pourcentages de limons fins obtenus quelle que soit la distance de prélèvement $(\mathrm{P} \geq 0,16)$. En revanche, il existe une variabilité entre les sites d'étude sur certaines distances. La valeur de limons fins notée à $20 \mathrm{~m}$ de $E$. camaldulensis à Boucotte Diembéring est plus élevée que celle mesurée dans les autres sites à la même distance de prélèvement $(\mathrm{P}<0,03)$ (Tableau 6).

Aucune différence significative n'a été observée entre la teneur en limons grossiers et en sables fins obtenues quelle que soit la distance de prélèvement et le site $(\mathrm{P} \geq 0,27)$ (Tableau 6). Cette teneur varie de $6,8 \%$ à $18.2 \%$ pour les limons grossiers et de $35,3 \%$ à $49,7 \%$ pour les sables fins et pour tous les traitements confondus. Quant à la teneur en sables moyens, l'analyse n'a montré aucune différence significative entre la teneur obtenue quelle que soit la distance de prélèvement $(P \geq 0,07)$. En revanche, cette teneur varie de façon différente entre les sites sur certaines distances. En effet, la teneur en sables moyens mesurée à $20 \mathrm{~m}$ de E. camaldulensis à Boucotte Diembéring est significativement plus importante que celle notée dans les autres sites d'étude. Cette teneur en sables moyens est comprise entre 22,3\% et $41,3 \%$. Il en est de même pour les sables grossiers. En effet, l'analyse n'a montré aucune différence significative entre la teneur en sables grossiers quelle que soit la distance de prélèvement $(\mathrm{P} \geq 0,062)$ (Tableau 6). Cependant, celle mesurée à $0 \mathrm{~m}$, à $20 \mathrm{~m}$ et à $30 \mathrm{~m}$ de E. camaldulensis à Oussouye est significativement plus élevée que la teneur obtenue dans les autres sites 
d'étude $(\mathrm{P}<0,023)$. Cette teneur en sables grossiers varie de $0,2 \%$ à $1,4 \%$ pour tous les traitements.

Tableau 6: Teneurs des caractéristiques physiques en fonction de la distance par rapport au pied de E. camaldulensis et des sites étudiés

\begin{tabular}{llcccc}
\hline Eléments physiques & \multicolumn{1}{c}{ Sites } & $\mathbf{0 ~} \mathbf{~ m}$ & $\mathbf{1 0} \mathbf{~ m}$ & $\mathbf{2 0} \mathbf{~ m}$ & $\mathbf{3 0 ~} \mathbf{~}$ \\
\hline \multirow{3}{*}{ Argiles \% } & Boucotte D & $8,6 \mathrm{~b}$ & $10,2 \mathrm{ab}$ & $11,2 \mathrm{ab}$ & $9,2 \mathrm{ab}$ \\
& Boukitingho & $9,9 \mathrm{a}$ & $7,9 \mathrm{a}$ & $10,3 \mathrm{a}$ & $12,0 \mathrm{a}$ \\
& Oussouye & $12,8 \mathrm{a}$ & $13,2 \mathrm{a}$ & $13,7 \mathrm{a}$ & $13,7 \mathrm{a}$ \\
\hline \multirow{3}{*}{ Limons Fins \% } & Boucotte D & $3,1 \mathrm{a}$ & $1,8 \mathrm{ab}$ & $0,9 \mathrm{~b}$ & $2,7 \mathrm{ab}$ \\
& Boukitingho & $1,9 \mathrm{a}$ & $4,3 \mathrm{a}$ & $3,1 \mathrm{a}$ & $2,3 \mathrm{ab}$ \\
& Oussouye & $3,5 \mathrm{a}$ & $3,3 \mathrm{a}$ & $4,1 \mathrm{a}$ & $3,1 \mathrm{a}$ \\
\hline \multirow{3}{*}{ Limons Grossiers \% } & Boucotte D & $9,3 \mathrm{a}$ & $18,2 \mathrm{a}$ & $6,8 \mathrm{a}$ & $8,7 \mathrm{a}$ \\
& Boukitingho & $15,9 \mathrm{a}$ & $6,7 \mathrm{a}$ & $8.0 \mathrm{a}$ & $14,6 \mathrm{a}$ \\
& Oussouye & $8,6 \mathrm{a}$ & $7,8 \mathrm{a}$ & $22,8 \mathrm{a}$ & $11,3 \mathrm{a}$ \\
\hline \multirow{3}{*}{ Sables Fins \% } & Boucotte D & $40,7 \mathrm{a}$ & $35,8 \mathrm{a}$ & $40,8 \mathrm{a}$ & $37,9 \mathrm{a}$ \\
& Boukitingho & $35,3 \mathrm{a}$ & $44,5 \mathrm{a}$ & $47 \mathrm{a}$ & $48,2 \mathrm{a}$ \\
& Oussouye & $49,7 \mathrm{a}$ & $48,7 \mathrm{a}$ & $38,2 \mathrm{a}$ & $45 \mathrm{a}$ \\
\hline \multirow{5}{*}{ Sables Moyens \% } & Boucotte D & $38,2 \mathrm{a}$ & $33,9 \mathrm{a}$ & $40 \mathrm{a}$ & $41,3 \mathrm{a}$ \\
& Boukitingho & $32,3 \mathrm{ab}$ & $31,7 \mathrm{ab}$ & $27,6 \mathrm{~b}$ & $22,3 \mathrm{ab}$ \\
& Oussouye & $24,9 \mathrm{ca}$ & $26,6 \mathrm{ca}$ & $19,8 \mathrm{c}$ & $26,5 \mathrm{ca}$ \\
\hline \multirow{3}{*}{ Sables Grossiers \% } & Boucotte D & $0,2 \mathrm{a}$ & $0,2 \mathrm{a}$ & $0,3 \mathrm{a}$ & $0,3 \mathrm{a}$ \\
& Boukitingho & $0,7 \mathrm{~b}$ & $0,6 \mathrm{~b}$ & $0,7 \mathrm{ab}$ & $0,7 \mathrm{~b}$ \\
& Oussouye & $0,51 \mathrm{c}$ & $0,46 \mathrm{c}$ & $1,4 \mathrm{ca}$ & $0,5 \mathrm{c}$ \\
\hline
\end{tabular}

Boucotte $\mathrm{D}=$ Boucotte Diembéring

Ces résultats ont montré en général que la distance de prélèvement par rapport à $E$. camaldulensis n'a pas d'effets significatifs sur les paramètres physiques du sol, quel que soit le site $(\mathrm{P} \geq 0,37)$. Une texture sablo-limoneuse a été notée, quelle que soit la distance de prélèvement par rapport à $E$. camaldulensis et le site d'étude.

Effet de la distance de prélèvement par rapport au pied de $E$. camaldulensis sur les propriétés chimiques du sol

Le $\mathrm{pH}$ mesuré varie entre 5,58 et 6,07 pour tous les traitements et quel que soit le site. La plus grande valeur est enregistrée à $10 \mathrm{~m}$ et $20 \mathrm{~m}$ de $E$. camaldulensis à Boukitingho $(6,07)$ (Tableau 7). 
Tableau 7: Variabilité des Caractéristiques chimiques du sol par rapport au pied de

\begin{tabular}{|c|c|c|c|c|c|}
\hline \multicolumn{6}{|c|}{ E. camaldulensis } \\
\hline Eléments chimiques & Sites & $\mathbf{0 ~ m}$ & $10 \mathrm{~m}$ & $20 \mathrm{~m}$ & $30 \mathrm{~m}$ \\
\hline \multirow{3}{*}{$\mathrm{pH}$ eau } & Boucotte D & $5,8 \mathrm{a}$ & 5,9 a & $6,1 \mathrm{a}$ & $5,8 \mathrm{a}$ \\
\hline & Boukitingho & $6,02 \mathrm{a}$ & $6,07 \mathrm{a}$ & $6,07 \mathrm{a}$ & $5,8 \mathrm{a}$ \\
\hline & Oussouye & $5,85 \mathrm{ab}$ & $5,63 \mathrm{~b}$ & $5,98 \mathrm{ab}$ & $5,58 \mathrm{~b}$ \\
\hline \multirow{3}{*}{$\mathrm{C} \%$} & Boucotte D & $0,3 \mathrm{a}$ & $0,22 \mathrm{a}$ & $0,27 \mathrm{a}$ & $0,26 \mathrm{a}$ \\
\hline & Boukitingho & $0,46 \mathrm{a}$ & $0,31 \mathrm{a}$ & $0,33 \mathrm{a}$ & $0,35 \mathrm{a}$ \\
\hline & Oussouye & $0,33 \mathrm{a}$ & $0,35 \mathrm{a}$ & $0,38 \mathrm{a}$ & $0,33 \mathrm{a}$ \\
\hline \multirow{3}{*}{ MO \% } & Boucotte D & $0,51 \mathrm{a}$ & $0,4 \mathrm{a}$ & $0,47 \mathrm{a}$ & $0,45 \mathrm{a}$ \\
\hline & Boukitingho & $0,79 \mathrm{a}$ & $0,53 \mathrm{a}$ & $0,56 \mathrm{a}$ & $0,61 \mathrm{a}$ \\
\hline & Oussouye & $0,56 \mathrm{a}$ & $0,6 \mathrm{a}$ & $0,66 \mathrm{a}$ & $0,57 \mathrm{a}$ \\
\hline \multirow{3}{*}{$\mathrm{N} \%$} & Boucotte D & $0,03 \mathrm{a}$ & $0,02 \mathrm{a}$ & $0,02 \mathrm{a}$ & $0,03 \mathrm{a}$ \\
\hline & Boukitingho & $0,039 \mathrm{a}$ & $0,027 \mathrm{a}$ & $0,026 \mathrm{a}$ & $0,03 \mathrm{a}$ \\
\hline & Oussouye & $0,027 \mathrm{a}$ & $0,03 \mathrm{a}$ & $0,026 \mathrm{a}$ & $0,028 \mathrm{a}$ \\
\hline \multirow{3}{*}{$\mathrm{C} / \mathrm{N}$} & Boucotte D & $11,9 \mathrm{a}$ & $11,6 \mathrm{a}$ & $13,7 \mathrm{a}$ & $11,6 \mathrm{a}$ \\
\hline & Boukitingho & $11,63 \mathrm{~b}$ & $11,52 \mathrm{~b}$ & $12,56 \mathrm{a}$ & $11,66 \mathrm{a}$ \\
\hline & Oussouye & $5,62 \mathrm{c}$ & $7,86 \mathrm{c}$ & $14,5 \mathrm{a}$ & $6,0 \mathrm{c}$ \\
\hline \multirow{3}{*}{ Ca meq/100g } & Boucotte D & $1,8 \mathrm{ac}$ & $1,8 \mathrm{a}$ & $1,6 \mathrm{ac}$ & $2,0 \mathrm{ac}$ \\
\hline & Boukitingho & $2,23 \mathrm{a}$ & $2,1 \mathrm{a}$ & $2,57 \mathrm{a}$ & $1,29 \mathrm{ac}$ \\
\hline & Oussouye & $1,33 \mathrm{c}$ & $1,28 \mathrm{c}$ & $2,36 \mathrm{a}$ & $1,4 \mathrm{c}$ \\
\hline \multirow{3}{*}{ Na meq/100g } & Boucotte D & $0,31 \mathrm{a}$ & $0,27 \mathrm{a}$ & $0,21 \mathrm{a}$ & $0,31 \mathrm{a}$ \\
\hline & Boukitingho & $0,25 \mathrm{~b}$ & $0,23 \mathrm{ab}$ & $0,21 a b$ & $0,23 a b$ \\
\hline & Oussouye & $0,22 \mathrm{~b}$ & $0,17 \mathrm{ab}$ & $0,15 a b$ & $0,28 \mathrm{ab}$ \\
\hline \multirow{3}{*}{$\mathrm{K}$ meq/100g } & Boucotte D & $0,3 \mathrm{a}$ & $0,26 \mathrm{a}$ & $0,21 \mathrm{a}$ & $0,31 \mathrm{a}$ \\
\hline & Boukitingho & $0,15 \mathrm{ab}$ & $0,13 \mathrm{a}$ & $0,11 \mathrm{a}$ & $0,16 \mathrm{a}$ \\
\hline & Oussouye & $0,1 \mathrm{a}$ & $0,12 \mathrm{a}$ & $0,11 \mathrm{a}$ & $0,13 \mathrm{a}$ \\
\hline \multirow{3}{*}{ P ppm } & Boucotte D & $9,5 \mathrm{a}$ & $10,5 \mathrm{a}$ & $6,75 \mathrm{a}$ & $7,25 \mathrm{a}$ \\
\hline & Boukitingho & $5,1 \mathrm{~b}$ & $5,23 \mathrm{~b}$ & $5,2 \mathrm{~b}$ & $3,03 \mathrm{~b}$ \\
\hline & Oussouye & $3,87 \mathrm{c}$ & $3,48 \mathrm{c}$ & $3,95 \mathrm{c}$ & $4,3 \mathrm{~b}$ \\
\hline \multirow{3}{*}{$\mathrm{Mg}$ meq/100g } & Boucotte D & $0,75 \mathrm{a}$ & $0,56 \mathrm{a}$ & $0,51 \mathrm{a}$ & $0,75 \mathrm{a}$ \\
\hline & Boukitingho & $0,9 \mathrm{a}$ & $0,96 \mathrm{a}$ & $0,86 \mathrm{a}$ & $0,77 \mathrm{a}$ \\
\hline & Oussouye & $0,8 \mathrm{a}$ & $0,6 \mathrm{a}$ & $0,9 \mathrm{a}$ & $0,9 \mathrm{a}$ \\
\hline \multirow{3}{*}{ CEC meq/100g } & Boucotte D & $24 \mathrm{a}$ & $23,25 \mathrm{a}$ & $21,75 \mathrm{a}$ & $23,8 \mathrm{a}$ \\
\hline & Boukitingho & $24 a b$ & $22,25 b$ & $22 \mathrm{a}$ & $24 \mathrm{a}$ \\
\hline & Oussouye & $23,75 \mathrm{ab}$ & $25,75 \mathrm{ab}$ & $22,75 \mathrm{a}$ & $26,25 \mathrm{a}$ \\
\hline
\end{tabular}

$\mathrm{C} \%=$ Pourcentage en Carbone, $\mathrm{MO} \%=$ Pourcentage en Matière organique, $\mathrm{N} \%=$ Pourcentage en Azote, $\mathrm{Na}=$ Sodium, $\mathrm{Ca}=$ Calcium, $\mathrm{K}=$ Potassium, $\mathrm{P}=$ Phosphore assimilable, $\mathrm{Mg}=$ Magnésium, $\mathrm{CEC}=$ Capacité d'échange cationique, Boucotte $\mathrm{D}=$ Boucotte Diembéring

Il ressort de l'analyse que le $\mathrm{pH}$ est modérément acide quelle que soit la distance de prélèvement par rapport à E. camaldulensis à Oussouye et 
Boucotte Diembéring, à l'exception du pH obtenu à $20 \mathrm{~m}$ de E. camaldulensis à Boucotte Diembéring qui est légèrement acide (6,07). A Boukitingho, les valeurs du $\mathrm{pH}$ mesuré à $0 \mathrm{~m}, 10 \mathrm{~m}$ et à $20 \mathrm{~m}$ de E. camaldulensis sont légèrement acide. Cependant à $30 \mathrm{~m}$ de E. camaldulensis, celui-ci est modérément acide. De façon globale, l'analyse montre un $\mathrm{pH}$ relativement acide au niveau des différents traitements et sites (Tableau 7).

Les teneurs en carbone organique $(\mathrm{C})$ et en matière organique $(\mathrm{MO})$ sont comprises entre $0,22 \%$ et $0,46 \%$ pour le carbone et de $0,40 \%$ à $0,79 \%$ pour la matière organique. La plus grande valeur de carbone $(0,46 \%)$ et de matière organique $(0,79 \%)$ est obtenue à $0 \mathrm{~m}$ de E. camaldulensis à Boukitingho. Le taux de la MO d'une fraction de sol est fonction de sa teneur en carbone organique (C). L'analyse statistique n'a montré aucune différence significative entre les valeurs de carbone et de la matière organique mesurées pour toutes les distances de prélèvement confondues avec respectivement $\mathrm{P} \geq 0,19$ pour le carbone et $\mathrm{P} \geq 0,14$ pour la matière organique. Globalement le sol est pauvre en carbone et en matière organique quelle que soit la distance de prélèvement et le site d'étude $(\mathrm{MO}<0,76 \%)$.

Aucune différence significative n'a été notée entre les valeurs d'azote (N) mesurées aussi bien pour les distances de prélèvement par rapport à $E$. camaldulensis que pour les sites $(\mathrm{P}<0,05)$. La teneur en azote des sols des différents traitements est comprise entre $0,02 \%$ et $0,039 \%$. Il ressort de l'analyse, que les valeurs de l'azote sont très faibles par rapport à la norme $(\mathrm{N}<0,8 \%)$. D'une manière générale, les teneurs en azote des sols prélevés traduisent un sol très pauvre quelle que soit la distance de prélèvement et le site.

Les valeurs de $\mathrm{C} / \mathrm{N}$ mesurés sont quant à elles comprises entre 5,62 et 14,5. L'analyse a montré une différence significative entre le rapport $\mathrm{C} / \mathrm{N}$ mesuré à Oussouye à $0 \mathrm{~m}, 10 \mathrm{~m}$ et à $30 \mathrm{~m}$ de $E$. camaldulensis comparé à celui qui est mesuré à $20 \mathrm{~m}$ de $E$. camaldulensis $(\mathrm{P}<0,05)$. Cette même tendance est observée à Boukitingho. En effet l'analyse de variance a révélé que le rapport $\mathrm{C} / \mathrm{N}$ obtenu à $20 \mathrm{~m}$ et à $30 \mathrm{~m}$, est plus élevé que celui qui est obtenu à $0 \mathrm{~m}$ de E. camaldulensis $(\mathrm{P}=0,03)$. En revanche aucune différence significative n'a été observée entre le $\mathrm{C} / \mathrm{N}$ obtenu à Boucotte Diembéring, quelle que soit la distance de prélèvement. Ces valeurs enregistrées prouvent de forts taux de minéralisation de la matière organique sous les différents traitements.

Pour ce qui est du calcium $(\mathrm{Ca})$, du sodium $(\mathrm{Na})$, du potassium $(\mathrm{K})$ et du magnésium $(\mathrm{Mg})$, l'analyse statistique n'a révélé aucune différence significative entre ces éléments quelle que soit la distance de prélèvement par rapport à E. camaldulensis et le site $(\mathrm{P}>0,05)$, à l'exception du calcium obtenu à $20 \mathrm{~m}$. En effet, le calcium obtenu à $20 \mathrm{~m}$ de E. camaldulensis à Boukitingho et à Boucotte Diembéring est significativement plus élevé que celui mesuré à Oussouye $(\mathrm{P}<0,02)$. Les valeurs de calcium mesurées sont comprises entre 1,4 
et 2,57 meq/100g pour tous les traitements et les sites confondus. Ces valeurs étant supérieur à $0,2 \mathrm{meq} / 100 \mathrm{~g}$, on observe alors une réponse non favorable aux engrais calciques. Quant au potassium, les valeurs obtenues sont comprises entre 0,1 et $0,31 \mathrm{meq} / 100 \mathrm{~g}$ pour tous les traitements et les sites confondus. Globalement, On observe alors une réponse favorables avec les engrais potassiques pour la majorité des traitements et des sites car ces valeurs étant inférieurs à $0,2 \mathrm{meq} / 100 \mathrm{~g}$, à l'exception du potassium mesuré à $0 \mathrm{~m}$ et à $30 \mathrm{~m}$ de E. camaldulensis à Boucotte Diembéring. Les valeurs du potassium obtenu à $0 \mathrm{~m}$ et à $30 \mathrm{~m}$ étant supérieures à $0,2 \mathrm{meq} / 100 \mathrm{~g}$, ces sols ne sont pas adaptés en engrais potassiques.

La teneur en sodium des sols mesurés est comprise entre 0,15 et 0,31 meq/100 g. Toutes ces valeurs du sodium sont inférieurs à $1 \mathrm{meq} / 100 \mathrm{~g}$ aussi bien pour les distances par rapport à E. camaldulensis que pour les sites. Celles-ci montrent que ces sols ne sont pas potentiellement sodiques. Quant à la teneur en magnésium, elle est supérieure ou égale à $0,5 \mathrm{meq} / 100 \mathrm{~g}$. Cette teneur est comprise entre 0,51 et $0,96 \mathrm{meq} / 100 \mathrm{~g}$ pour tous les traitements et les sites confondus. Elles traduisent donc que les sols n'ont une carence en magnésium car étant supérieur ou égale à $0,5 \mathrm{meq} / 100 \mathrm{~g}$ quelle que soit la distance de prélèvement par rapport à E. camaldulensis et le site.

L'analyse de variance montre aucune différence significative entre le phosphore assimilable mesuré, quelle que soit la distance de prélèvement $(\mathrm{P}>0,05)$. En revanche celui obtenu à Boucotte Diembéring est significativement différent de ceux mesurés à Oussouye et à Boukitingho. La plus grande valeur est enregistrée à $20 \mathrm{~m}$ de E. camaldulensis à Boucotte Diembéring. La teneur en phosphore est comprise entre 3,03 et 10,5ppm pour tous les traitements et les sites. De façon générale, ces valeurs étant supérieurs à 5 ppm, montre que la tenure en phosphore est basse, c'est ce qui traduit une réponse très probable de ces sols aux engrais.

L'analyse de variance ne montre aucune différence significative entre la CEC mesurée quelle que soit la distance par rapport à E. camaldulensis (P>0,05). La valeur de la CEC est comprise entre 21,75 et 26,25 meq/100g. Ces valeurs de la CEC, montrent que les éléments nutritifs contenus dans le sol prélevé à différentes distances par rapport à E. camaldulensis, sont potentiellement disponibles pour d'éventuelles cultures.

En somme, il ressort de ces analyses, que la distance de prélèvement par rapport à E camaldulensis n'a pas d'effets significatifs sur les propriétés chimiques du sol. Les caractéristiques chimiques du sol de toutes les distances confondus par rapport à E. camaldulensis, ne semblent pas être influencées par l'espèce, quelle que soit la distance et quel que soit le site $(\mathrm{P}>0,05)$ (Tableau 7). 


\section{Corrélation entre les éléments physico-chimiques du sol et la distance de} prélèvement par rapport au pied de $E$. camaldulensis

L'analyse en Composantes Principales (ACP) a permis de montrer que les paramètres comme le carbone, la matière organique, l'azote, le sodium, le potassium, la capacité d'échange cationique sont situés du côté des abscisses positives (axe 1) et plus corrélés à la distance de prélèvement de $0 \mathrm{~m}$ et $30 \mathrm{~m}$ du collet de E. camaldulensis (Fig.7). Ce qui signifie que les valeurs de ces paramètres sont plus importantes pour ce traitement comparé aux autres paramètres. Il en est de même pour le calcium, les limons, le pH du sol, l'argile et le rapport $\mathrm{C} / \mathrm{N}$, qui sont tous situés du côté des ordonnées négatives et qui sont plus corrélés à la distance de $20 \mathrm{~m}$ par rapport au collet de $E$. camaldulensis.

Il faut également noter que le sable et le phosphore total en pourcentage sont plus ou moins proches de l'origine de l'axe des abscisses et de l'axe des ordonnées. Cela signifie que ces paramètres ne changent pas beaucoup suivant les différentes distances.

Suivant les axes, la figure peut être subdivisée en trois (3) groupes : - le premier groupe corrélé à la distance $0 \mathrm{~m}$ par rapport au pied de $E$. camaldulensis. Ce groupe est caractérisé par une matière organique élevé, un taux de carbone élevé, la présence de magnésium et d'azote issu de la minéralisation de la matière organique.

- le deuxième groupe est corrélé à la distance $30 \mathrm{~m} \mathrm{du}$ pied de $E$. camaldulensis. Ce groupe est caractérisé par la présence du potassium, du sodium et une capacité d'échange cationique, un rapport $\mathrm{C} / \mathrm{N}$ faible.

- $\quad$ et le troisième groupe est corrélé à la distance $20 \mathrm{~m}$ du pied de $E$. camaldulensis. Ce groupe s'oppose au groupe 2 et se caractérise par un rapport $\mathrm{C} / \mathrm{N}$ élevé, par la présence de limons et d'argile et du calcium, un pH élevé. 


\section{Biplot (axes F1 and F2: $82.22 \%$ )}

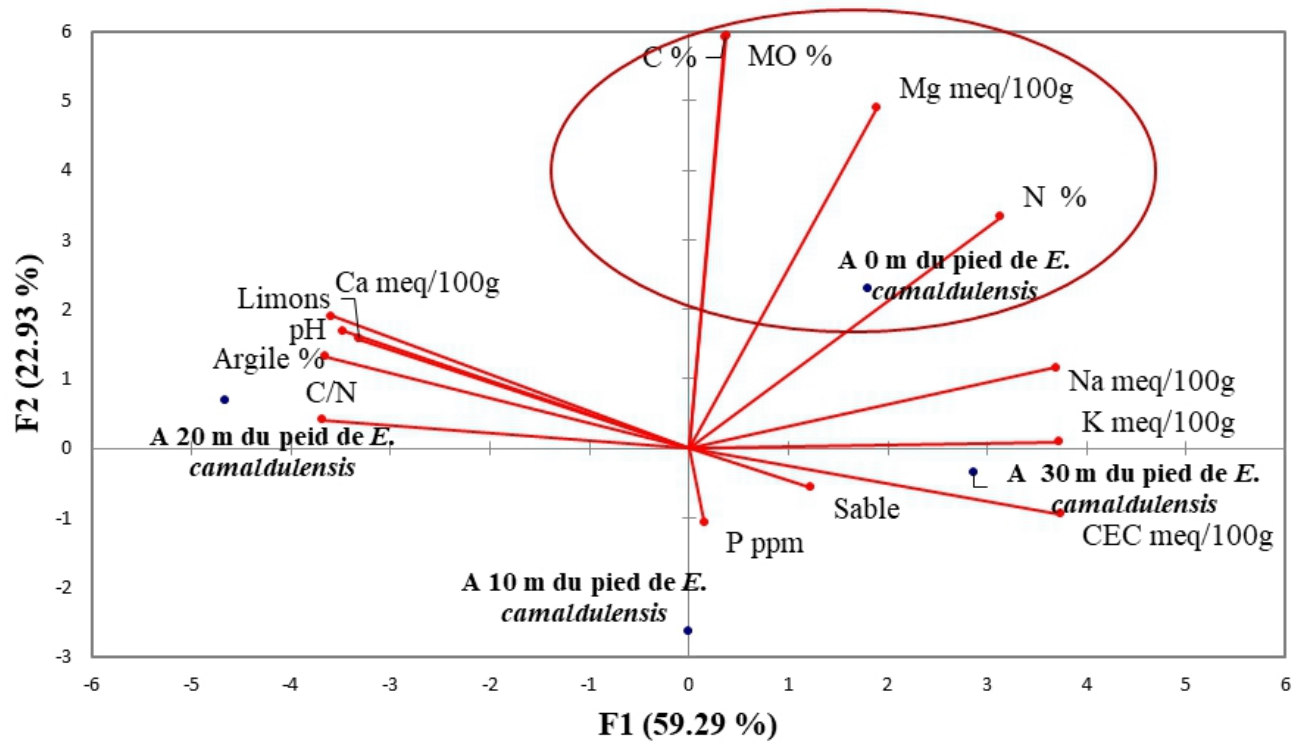

Figure 7: Corrélation entre les paramètres physico-chimiques du sol et la distance de prélèvement par rapport au pied de E. camaldulensis

$\mathrm{C} \%=$ Pourcentage en carbone, $\mathrm{MO} \%=$ Pourcentage en matière organique, $\mathrm{Mg}=$ Magnésium,

$\mathrm{N} \%=$ Pourcentage en Azote, $\mathrm{Na}=$ Sodium, $\mathrm{K}=$ Potassium, $\mathrm{CEC}=$ Capacité d'échange cationique, $\mathrm{P}_{\mathrm{ppm}}=$ Phosphore en ppm, $\mathrm{Ca}=$ Calcium, $\mathrm{C} / \mathrm{N}=$ Rapport carbone sur Azote.

\section{Discussion}

Les résultats de l'analyse statistique des données sur les propriétés physiques du sol n'ont montré aucune différence significative, quelle que soit la distance de prélèvement et quel que soit le site, à l'exception du pourcentage des limons fins mesurés à $0 \mathrm{~m}$ de E. camaldulensis à Boukitingho. Ces résultats montrent qu'en général la distance de prélèvement par rapport à $E$. camaldulensis, n'a pas d'effets significatifs sur les paramètres physiques du sol, quelle que soit la distance par rapport à E. camaldulensis et quel que soit le site $(\mathrm{P} \geq 0,37)$. La texture du sol est sablo-limoneuse, quelle que soit la distance de prélèvement par rapport à E. camaldulensis et le site. La similarité des caractéristiques physiques du sol pourrait être due à des conditions climatiques favorables de la zone. Ce résultat est conforme à celui de Akpo (1998), qui a montré qu'à l'exception des teneurs en sables grossiers qui indiquent une légère diminution depuis la zone située près du tronc à l'extérieur de l'ombrage, l'arbre n'agit pas sur les autres fractions granulométriques. Il apparaît surtout un effet profondeur: un enrichissement en argile. L'appauvrissement en limon est peu perceptible.

Il ressort de l'analyse des propriétés chimiques que le $\mathrm{pH}$ est modérément acide quelle que soit la distance de prélèvement par rapport à $E$. 
camaldulensis à Oussouye et Boucotte Diembéring, à l'exception du $\mathrm{pH}$ obtenu à $20 \mathrm{~m}$ de $E$. camaldulensis à Boucotte Diembéring qui est légèrement acide $(6,07)$. Le $\mathrm{pH}$ mesuré varie entre 5,58 et 6,07 pour tous les traitements et quel que soit le site. Ces résultats sont conformes à ceux obtenus par Abdou et al., (2014) qui avaient trouvé une différence non significative entre les valeurs du $\mathrm{pH}$ sous et hors houppier. Hailu et al., (2003) ont révélé également aucune différence significative de l'acidité entre le site d'implantation de $E$. globulus et les terres agricoles adjacentes. Ce résultat pourrait être dû à la densité des plantations étudiées qui n'est pas forte.

Les résultats montrent qu'aucune différence significative n'a été notée entre l'azote $(\mathrm{N})$, la matière organique mesurées quelle que soit la distance de prélèvement par rapport à E. camaldulensis et quel que soit le site. Ce résultat confirme ceux de Saidou et al., (2018) qui ont trouvé aucune différence significative entre la matière organique et l'azote trouvés entre la zone couverte et non couverte par A. senegal. Ce résultat pourrait être dû par le faible apport en litière des plantes de E .camaldulensis dans la zone. Aucune différence significative dans les teneurs en azote $(\mathrm{N})$, tant entre sous et hors que selon l'éloignement au tronc de l'arbre (Akpo 1998).

L'analyse n'a montré globalement aucune différence significative entre le rapport $\mathrm{C} / \mathrm{N}$ mesuré quelle que soit la distance et quel que soit le site E. camaldulensis. Ces résultats sont en désaccord avec ceux obtenus par Githae et al. (2011) qui a trouvé un ratio plus élevé hors houppier d'A. senegal que sous son houppier. Cependant, Saidou et al., (2018) ont montré aucune différence significative entre le $\mathrm{C} / \mathrm{N}$ trouvé entre la hors et sous houppier. Il est aussi conforme à celui de Akpo (1998), qui a montré que sous Parkia biglobosa, le rapport $\mathrm{C} / \mathrm{N}$ est plus faible près du pied et plus élevé à la fin du couvert, tout au moins dans les deux premiers niveaux. Hors arbre, généralement, moins important, il croît en revanche avec la profondeur.

Aucune différence significative n'a été notée entre le Phosphore assimilable mesuré, quelle que soit la distance de prélèvement. Ces résultats sont en désaccord avec ceux obtenus par El Atta et al. (2013), sur plusieurs espèces d'Acacia et Bazongo et al. (2015) sur d'autres espèces comme Detarium microcarpa et Jatropha curcas.

L'analyse statistique a révélé aucune différence significative entre le calcium $(\mathrm{Ca})$, le Sodium $(\mathrm{Na})$, le Potassium $(\mathrm{K})$, le Magnésium $(\mathrm{Mg})$ mesurés quelle que soit la distance de prélèvement par rapport à E. camaldulensis et le site d'étude. Ce résultat infirme ceux de Farley et al., (2008) qui ont montré une acidification des eaux de surface liée à l'appauvrissement du sol en cations sur des bassins versants où les pâturages ont été remplacés par des plantations de Eucalyptus. Tchawa et Demaze (2002) ont montré un enrichissement des sols sous E. camaldulensis en base échangeables (magnésium, calcium et potassium). Soumaré (2012) a montré, également, un résultat semblable dans 
un site expérimental en Uruguay, dix ans après le remplacement de la végétation locale par une plantation de Eucalyptus. Kisa et al., (2007) ont montré également des résultats similaires concernant les espèces exotiques telles que Gmelina arborea et E. Camaldulensis, respectivement. Ces auteurs ont montré que ces espèces exotiques changent la structure et la diversité fonctionnelle.

\section{Conclusion}

Cette étude a permis d'évaluer l'effet de E. camaldulensis sur les propriétés physico-chimiques du sol. Les résultats de l'étude n'ont montré aucune différence significative entre l'argile, les limons fins et grossiers, les sables fins et grossiers mesurés, quelle que soit le distance de prélèvement par rapport à E. camaldulensis et quel que soit le site. Cependant le pourcentage de sable moyen obtenu à $10 \mathrm{~m}$ et de limons fins obtenu à $0 \mathrm{~m}$ de $E$. camaldulensis sont significativement plus élevés que ceux trouvés dans les autres distances de prélèvement.

Les résultats des analyses chimiques ont montré globalement, un $\mathrm{pH}$ modérément acide quelle que le la distance et quel que soit le site. Ils ont également montré que le sol est pauvre en carbone et matière organique quelle que soit la distance de prélèvement et quel que soit le site. Les teneurs en azote des sols prélevés traduisent un sol très pauvre quelle que soit la distance de prélèvement et quel que soit le site. Les caractéristiques chimiques du sol à différents distances de E. camaldulensis ne semblent pas être influencées par l'espèce, quelle que soit la distance et quel que soit le site.

\section{References:}

1. Abdou, M. M., Zoubeirou, A. M., Dan Lamso, N., et Ambouta, J. M.K. (2014). Productivité de la culture du sorgho (Sorghum bicolor) dans un système agroforestier à base d'Acacia senegal (L.) Willd. Au Niger. Journal of Applied Biosciences 82:7339-7346.

2. Akpo, L.E. (1998). Effet de l'arbre sur la végétation herbacée dans quelques phytocénoses au Sénégal variation selon un gradient climatique, Thèse de doctorat d'état en SCIENCES NATURELLES, $p$ 133.

3. Badji, M. (2016). Caractérisation et évaluation de la biomasse ligneuse et de la séquestration du carbone dans les plantations de Eucalyptus camaldulensis Dehn., du projet CBED dans le département d'Oussouye. Mémoire de Master, Université Assane Seck de Ziguinchor, p. 59

4. Bazongo, P., Traore, K., Traore, O., Yelemou, B., Sanon, K.B., Kabore, S., Victor Hien, V., et Nacro, B.H. (2015). Influence des haies de Jatropha sur le rendement d'une culture de sorgho (Sorghum 
vulgare) dans la zone Ouest du Burkina Faso: cas du terroir de Torokoro .Int. J. Biol. Chem. Sci. 9(6): 2595-2607.

5. Bray, R.H., et Kurtz, L.T. (1945). Determination of total organic and available forms of phosphorus in soils. Soil Sci., N059, pp.39-45.

6. Brechet, L., Ponton, S., Roy, J., Freycon, V., Couteaux, M.M., Bonal, D., Epron, D. (2009). "DO tree species characteristics influence soil respiration in tropical forests a test based on 16 tree species planted in monospecific plots. Plant and soil 319, 235-246.

7. CIRARD et GRET (2002).- Mémento de l'agronome, Ministère des Affaires Etrangères, France, p 1700.

8. COMIFER (2009). Teneurs en phosphore, potassium et magnésium des organes végétaux récoltés. [En ligne], Mise en ligne en novembre 2009, Consulté le 7 September 2017, URL : http://www.comifer.asso.fr/index.php/groupes-de-travail/pk-etmg.html

9. El Atta, H., Aref, I., Ahmed, A. (2013). Effect of Acacia spp. on soil properties in the highlands of Saudi Arabia. Life Science Journal; 10 (4).

10. Farley, K.A., Pineiro, G., Palmer, S., Jobbagy, E., Jackson, R. (2008). Stream acidification and base cation losses with grassland afforestation. Water Resources Research p 44.

11. GIEC (2007). Bilan 2007 des changements climatiques. Contribution des groupes de travail I, II et III au quatrième rapport d'évaluation du groupe d'experts intergouvernemental sur l'évolution du climat. (Pachauri R.K., Reisinger A., eds.) GIEC, Genève, Suisse, 103 p.: 911.

12. Githae, E.W., Gachene, C.K., Jesse, T., \& Njoka, J. T. (2011). Soil physicochemical properties under Acacia senegal varieties in the dryland areas of Kenya. Afr. J. Plant Sci.5 (8), p. 475-482

13. Hailu, A., and Veeman, T.S., (2003). "Comparative Analysis of Efficiency and Productivity Growth in Canadian Regional Boreal Logging Industries.” Canadian J Forest Research 33(9): 1653-1660

14. Hillebrand, W.F., Lundell, G.E.F., Bright, H.A., Hoffman, J.I. (1953). Applied Inorganic analysis, 2nd ed. John Wiley and Sons, Inc., NewYork, USA, 1034p

15. Keller, M., and Zengler K. 2004. Tapping into microbial diversity. Nature Reviews Microbiology 2(2):141-150.

16. Kisa, M., Sanon, A., Thioulouse, J., Assigbetse, K., Sylla, S., Spichiger, R., Dien, L., Berthelin, J., Prin, Y., Galiana, A., Lepage, M., Duponnois, R. (2007). Arbuscularmycorrhizal symbiosis can counterbalance the negative influence of the exotic tree species Eucalyptus camaldulensis on the structure and functioning of soil 
microbial communities in a sahelian soil. FEMS Microbiology Ecology 62, 32-44.

17. Landon, J.R. (1984)- Booker Tropical Soil Manual. xiv. Booker Agriculture International Ltd., London, and Longman, Burnt Mill, U.K, p 450.

18. Masson, J.C. (2012).- Etude du sol de la région d'Aigrefeuille-surMaine (Loire-Atlantique) à partir de la base de données des analyses de terre (INRA) ; Donesol, $18 \mathrm{p}$.

19. Orwin, K.H., Wardle, D.A. (2005). Plant species composition effects on belowground properties and the resistance and resilience of the soil microflora to drying disturbance. Plant and soil 278, 205-221.

20. Sagna, P. (2005). Dynamique du climat et son évolution récente dans la partie ouest de l'Afrique occidentale. Thèse, Université Cheikh Anta DIOP de Dakar, p 790.

21. Saidou, A.K., Hassane, M., Abdoullatif, Y T., Karimou, A. (2018). Effets d'Acacia senegal (L.) Willd sur le Rendement du Niébé (Vigna unguiculata) au Niger, Afrique de l'ouest, European Scientific Journal September 2018 edition Vol.14, No.27 ISSN: 1857 - 7881 (Printe)ISSN 1857- 7431 URL:http://dx.doi.org/10.19044/esj.2018.v14n27.p 176.

22. Soumare, A. (2012). Impact de Eucalyptus camaldulensis sur la diversité génétique et fonctionnelle des communautés microbiennes telluriques, les associations symbiotiques rhizobienne et endomycorhizienne et le développement de quelques légumineuses sahéliennes. Thèse, Université Cheikh Anta Diop de Dakar. p 244.

23. Tchawa, P., Demaze, M.T. (2002). Gestion de l'espace et effets écologiques de l'eucalypculture en pays Bamiléké (Ouest Cameroun) : stratégie paysanne et prise en compte d'un risque perçu, Revue de géographie de Bordeaux, ISSN : 1961-8603, DOI : 10.4000/com.108 